\title{
Corrigendum
}

\section{Corrigendum to "The Relationship between Population T4/TSH Set Point Data and T4/TSH Physiology"}

\author{
Stephen Paul Fitzgerald ${ }^{1,2}$ and Nigel Geoffrey Bean ${ }^{3,4}$ \\ ${ }^{1}$ Department of Internal Medicine and Department of Endocrinology, The Royal Adelaide Hospital, Adelaide, SA 5000, Australia \\ ${ }^{2}$ School of Medicine, The University of Adelaide, Adelaide, SA 5005, Australia \\ ${ }^{3}$ School of Mathematical Sciences, The University of Adelaide, Adelaide, SA 5005, Australia \\ ${ }^{4} A R C$ Centre of Excellence for Mathematical and Statistical Frontiers, The University of Adelaide, Adelaide, SA 5005, Australia
}

Correspondence should be addressed to Stephen Paul Fitzgerald; stephen.fitzgerald2@sa.gov.au

Received 8 May 2017; Accepted 17 May 2017; Published 20 August 2017

Copyright (c) 2017 Stephen Paul Fitzgerald and Nigel Geoffrey Bean. This is an open access article distributed under the Creative Commons Attribution License, which permits unrestricted use, distribution, and reproduction in any medium, provided the original work is properly cited.

In the article titled "The Relationship between Population T4/TSH Set Point Data and T4/TSH Physiology," [1] there was an error in Figure 2(b), which should be corrected as shown in Figure 2.

Additionally, there were errors in Results, where the statement "The slope $(-0.6)$ of the population curve in the normal range [5] is similar to the slope $(-0.59)$ of the curve of physiological TSH studies [2], and therefore it would seem more likely that the slope there has been generated by the mechanism described in Figure 4(a), that is, the different degrees of variation in organ sensitivity" should be corrected to "The slope $(-0.06)$ of the population curve in the normal range [5] is similar to the slope $(-0.059)$ of the curve of physiological TSH studies [2], and therefore it would seem more likely that the slope there has been generated by the mechanism described in Figure 4(a), that is, the different degrees of variation in organ sensitivity."

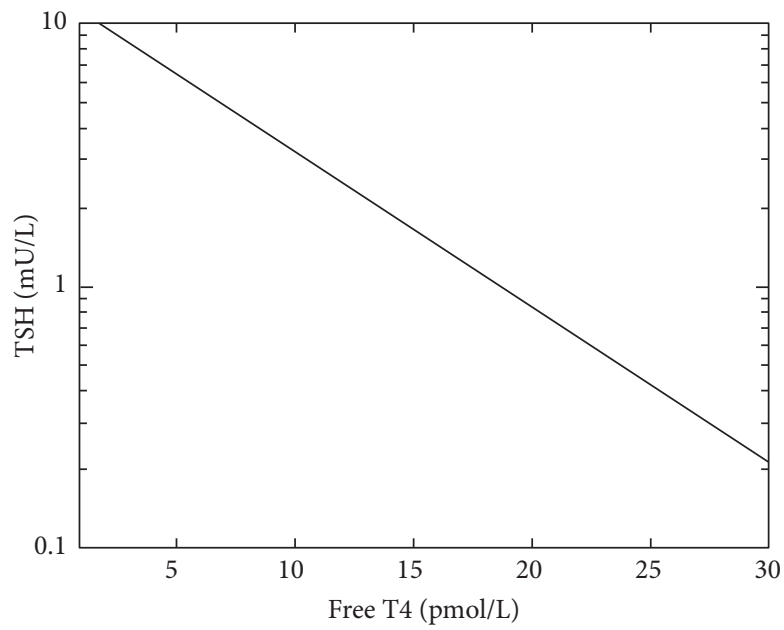

(a) TSH response to free T4

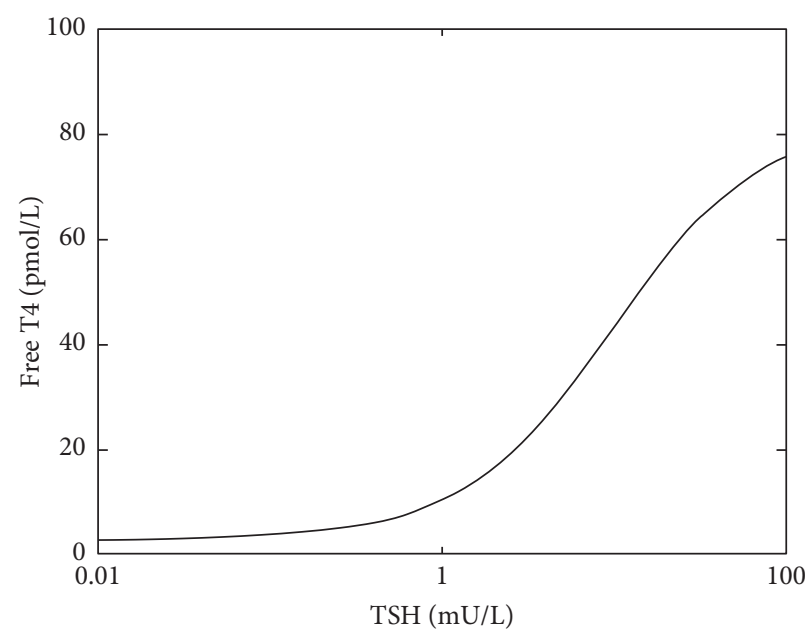

(b) Free $\mathrm{T} 4$ response to TSH

FIgURE 2: Continued. 


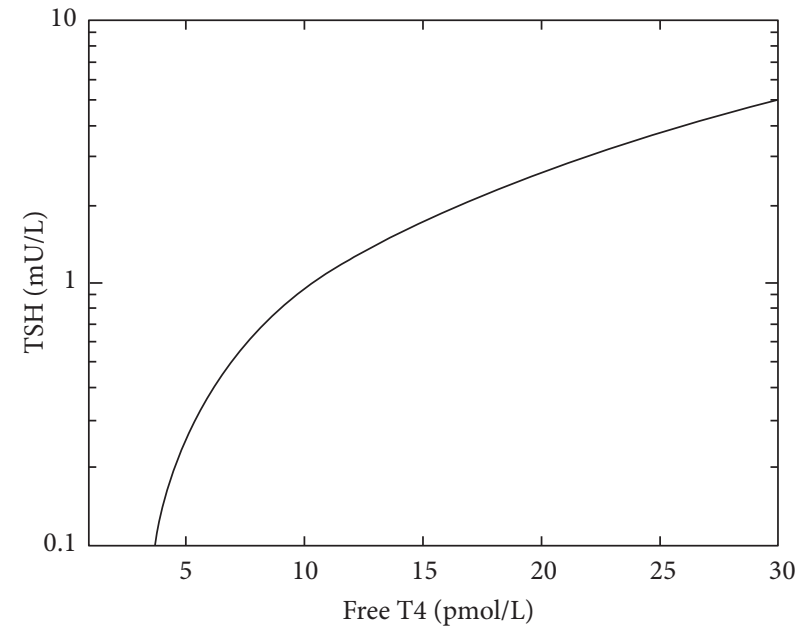

(c) Free T4 response to TSH

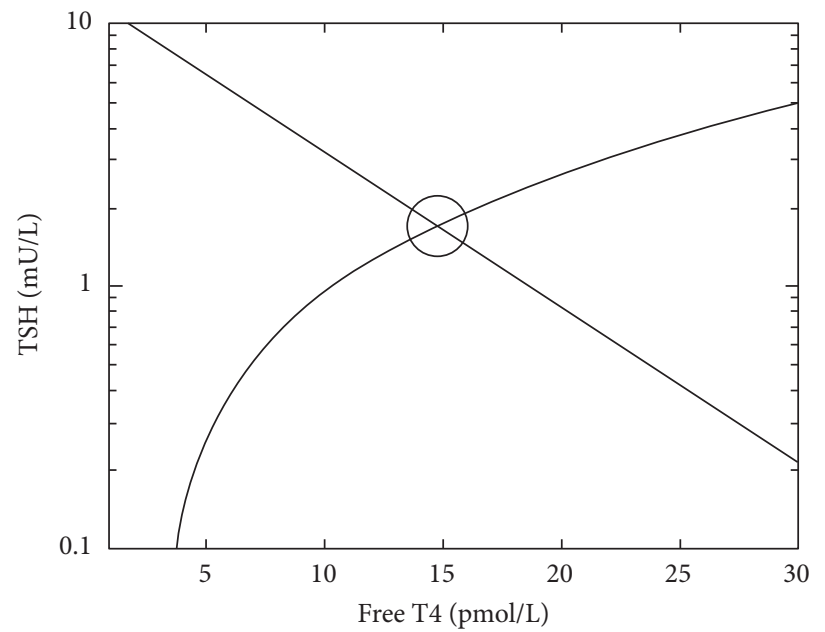

(d) Free T4/TSH levels

FIgure 2: (a) The TSH curve, (b, c) the T4 curve (on different axes, $K_{\mathrm{T}}$ approx. 41), and (d) the location of the T4/TSH set point.

\section{References}

[1] S. P. Fitzgerald and N. G. Bean, “The relationship between population T4/TSH set point data and T4/TSH physiology," Journal of Thyroid Research, vol. 2016, Article ID 6351473, 7 pages, 2016. 


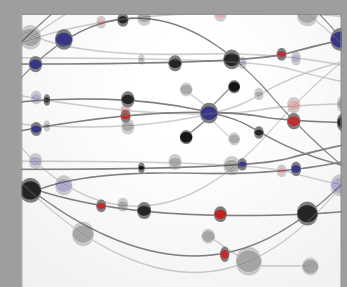

The Scientific World Journal
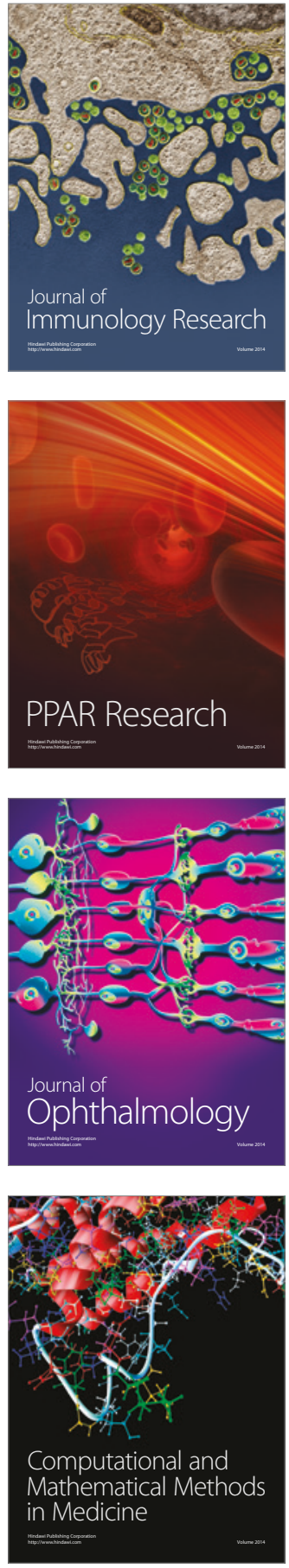

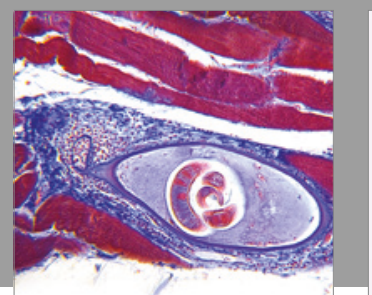

Gastroenterology Research and Practice
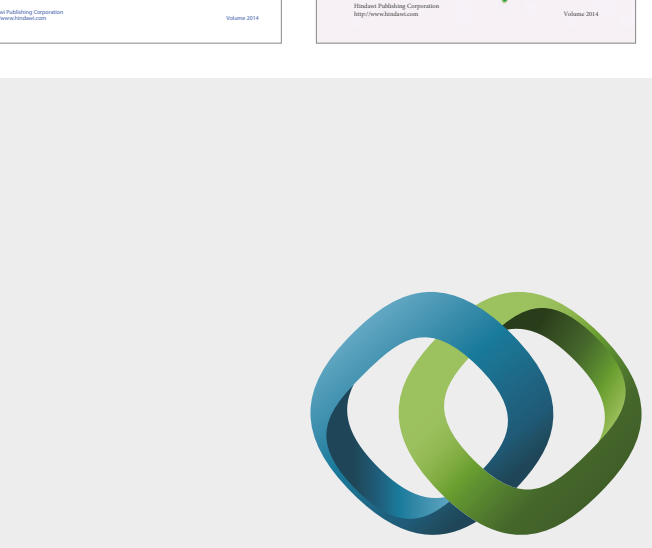

\section{Hindawi}

Submit your manuscripts at

https://www.hindawi.com
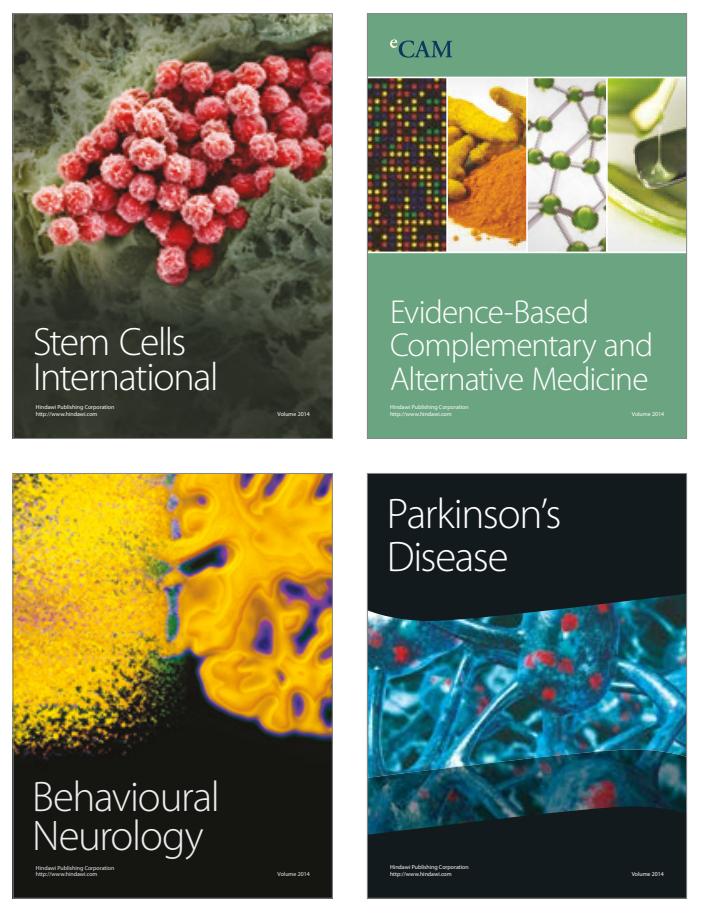
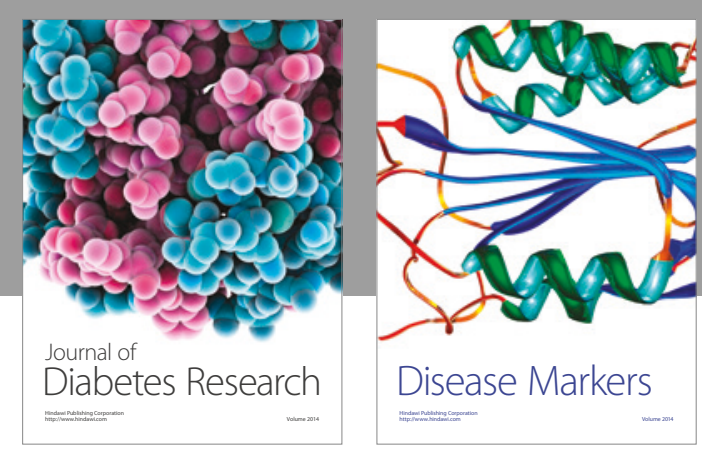

Disease Markers
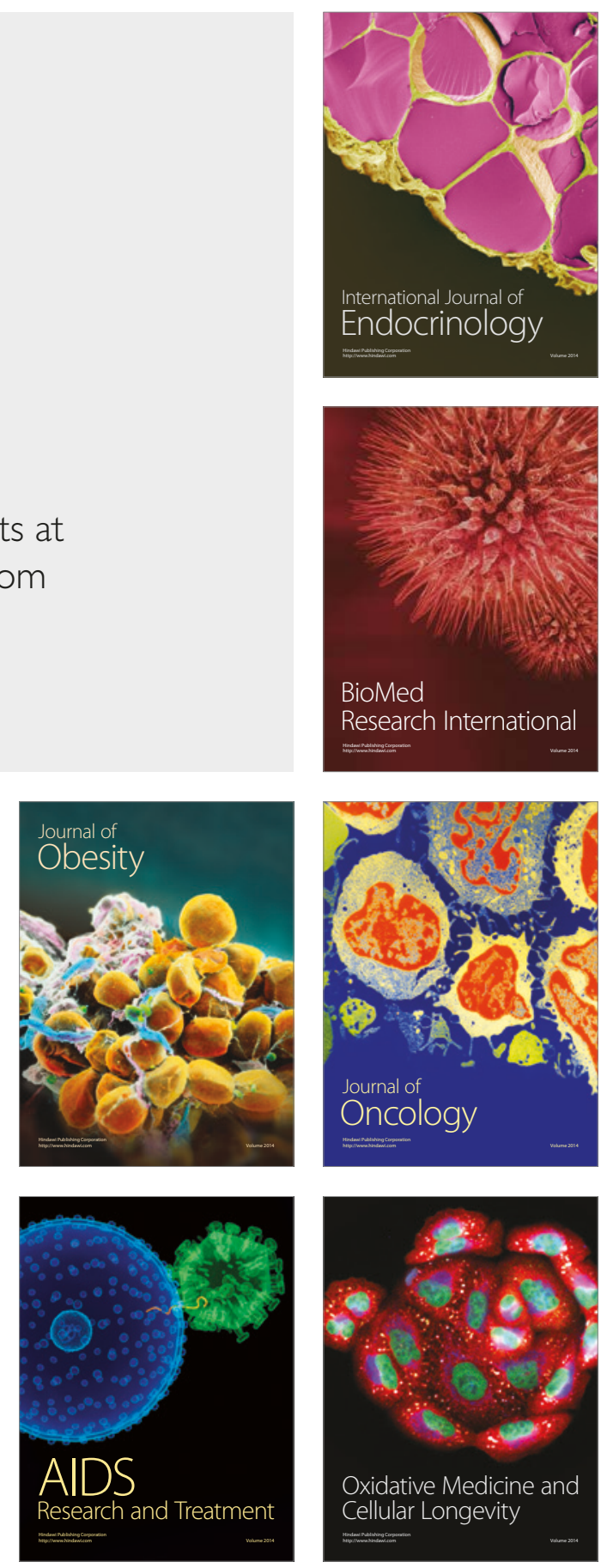